\title{
Power System Transient Stability Assessment Based on Quadratic Approximation of Stability Region
}

\author{
Ancheng Xue, Felix F. Wu, Fellow, IEEE, Yixin Ni, Senior Member, IEEE, Qiang Lu, Fellow, IEEE, \\ and Shengwei Mei, Member, IEEE
}

\begin{abstract}
This paper presents an approach to estimate the Critical Clearing Time (CCT) of the multi-machine power systems based on the quadratic surface which approximates the boundary of stability region relating to the controlling unstable equilibrium point. A decomposition method is developed to obtain the coefficients of the quadratic approximation surface. The CCT is determined by the crossing point of the quadratic surface and the continuous faulted trajectory. Simulations in IEEE 9-bus and New England system show the effectiveness of the proposed approach.
\end{abstract}

Index Terms-Transient Stability Assessment, Stability Region, Quadratic Approximation, Controlling Unstable Equilibrium point, Critical Clear Time

\section{INTRODUCTION}

T $\mathrm{N}$ the power system transient stability assessment (TSA), the concept of stability region (attraction region) and Controlling Unstable Equilibrium Point (CUEP) has been well recognized [1-6]. The boundary of the stability region for the power systems are composed of the stable manifolds of the unstable equilibrium point on the boundary [7], the CUEP is the unstable equilibrium point whose stable manifold is crossed by the continuous faulted trajectory. The CCT is determined by the crossing point of the stable manifold of the CUEP and the continuous faulted trajectory. Thus the description of the stable manifold of the CUEP plays an important role in TSA, various approximations to the stable manifold have been proposed. One way is to use the equal energy surface[8]-[13], which is determined by certain transient energy function, these approximations may always give out good results but have limitation for the non-existence

This work is supported by National Key Basic Research Special Fund of China (G2004CB217902), National Science Foundation of China (No.50377018,No.50595411) and Research Grant Council of HKSAR(HKU7176/03E).

Ancheng Xue is with the Department of Electrical engineering, Tsinghua University, Beijing 100084, China and the Graduate School at Shenzhen, Tsinghua University, Shenzhen 518005 China. (e-mail: xueac97@mails.tsinghua.edu.cn).

Felix $\mathrm{F}$. Wu and Yixin $\mathrm{Ni}$ are with the University of Hong Kong, Hong Kong, China. (e-mail: ffwu@eee.hku.hk, yxni@eee.hku.hk).

Qiang $\mathrm{Lu}$ and Shengwei Mei are with the Department of Electrical engineering, Tsinghua University, Beijing 100084, China. (e-mail: luqiang@mail.tsinghua.edu.cn, meishengwei@mail.tsinghua.edu.cn ). of transient energy function for general power system models. Another way is to obtain truncated approximations by applying the Taylor series expansion or the normal form method to the partial differential equation describing the stable manifold of the CUEP. With the idea of Taylor series expansion, ref. [14] obtained the hyper-plane approximation using the first order term and ref. [15] derived a hyper-surface approximation using the second order term. Recent work [16] also presented a quadratic approximation, but it lacks theoretical justification and need the energy correction procedure. Based on the extension of Poincare's classical result on normal formal theory to approximate the stable manifold, the early work [17] first proposed an algorithm to compute relevant coefficient of stable manifold in power series presentation. The work [18] originally used the normal form to compute the boundary of stability region, it got the exact series representation of the stable manifolds characterizing the stability boundary. This series can be computed recursively. However, no numerical tests have been conducted. With the real normal form, ref. [19] obtained the second order approximation for the stability region boundary, but this method requires the computation for all the eigenvectors and eigenvalues of the Jacobian matrix, which results in burdensome computation. To improve the normal form computation, ref. [20] presented another method to calculate the quadratic approximation based on similarity transformation which avoiding the computation for all the eigenvectors, unfortunately, this approximation is not correct [21]. The above normal form approximations are based on the implicit equation which describing the stable manifold in a new coordinate. Recently, Cheng and Ma et al. [21,22] discovered the explicit equation which describing the stable manifold of type- 1 equilibrium point in the original coordinate. Furthermore, they presented a quadratic approximation for the stable manifold of CUEP, which is the same as the hypersurface proposed in [15]. This paper applies the proposed quadratic approximation to determine the CCT of the multimachine power systems with the network-reduced structure. While obtaining the coefficients of quadratic approximation, we explore the special structure of the model and develop the decomposition method to reduce the computation. The estimated CCT is determined by the crossing point of the quadratic surface and the continuous faulted trajectory. The simulations in IEEE 9-bus and New England system show the 
effectiveness of the proposed approach.

The remainder of the paper is organized as following: Section II presents the quadratic approximation for the stable manifold of type-1 equilibrium point. Section III develops the decomposition approach to calculate the coefficients of the quadratic approximation to the stable manifold of CUEP for the multi-machine power systems which having the network reduction model and uniform damping. Section IV applies the proposed approach to different systems. Section V gives out the conclusions.

\section{Quadratic Approximation OF Stability Region}

Consider a nonlinear autonomous dynamic system described by the following differential equation defined on a manifold $M$,

$$
\dot{\boldsymbol{x}}=\boldsymbol{f}(\boldsymbol{x})
$$

where $\boldsymbol{f}, \boldsymbol{x} \in R^{m}, \boldsymbol{f} \in C^{2}$. An equilibrium point is a solution of the equation $f(x)=0$. A hyperbolic equilibrium point is an equilibrium point at which the Jacobian $D_{x} f$ has no zero real part eigenvalue. A type-k equilibrium point is a hyperbolic equilibrium point at which $k$ eigenvalues of $D_{x} f$ are having positive real parts. A hyperbolic stable equilibrium point $\boldsymbol{x}_{s}$ of system (1) is an equilibrium point at which all the eigenvaules of $D_{x} f$ (the Jacobian of the vector field) have negative real parts. The flow (or the solution with an initial state $\boldsymbol{x}$ ) of system (1) is expressed as:

$$
\varphi_{t}(\boldsymbol{x})=\varphi(\boldsymbol{x}, t)
$$

The stable manifold of a hyperbolic equilibrium point $\boldsymbol{x}_{0}$ is the set of all those points from each of which the flow will converge to $\boldsymbol{x}_{0}$ as time approaches positive infinite, i.e.

$$
W^{s}\left(x_{0}\right)=\left\{x \mid \lim _{t \rightarrow \infty} \varphi(x, t)=x_{0}\right\}
$$

If $\boldsymbol{x}_{s}$ is a hyperbolic stable equilibrium point for the system $V 1 \mathrm{VI}$, then $V\left(\boldsymbol{x}_{s}\right)=W^{s}\left(\boldsymbol{x}_{s}\right)$ is the stability region of the hyperbolic stable equilibrium point $\boldsymbol{x}_{s}$.

Under certain hyperbolic assumption for the autonomous system (1), the boundary of the stability region $\partial V\left(\boldsymbol{x}_{s}\right)$ is composed of the stable manifolds of the unstable equilibrium points on the boundary of the stability region [5], that is

$$
\partial V\left(\boldsymbol{x}_{s}\right)=\bigcup_{x_{e} \in \partial V} W^{s}\left(\boldsymbol{x}_{e}\right)
$$

Furthermore, the stable manifold of a type- 1 equilibrium point $\boldsymbol{x}_{e}$ can be described as following set $[21,22]$

$$
W^{s}\left(\boldsymbol{x}_{e}\right)=\left\{\begin{array}{l}
\boldsymbol{x} \mid \begin{array}{l}
h(\boldsymbol{x})=0, h\left(\boldsymbol{x}_{e}\right)=0 \\
f^{T} \cdot D_{x} h=\mu \cdot h
\end{array}
\end{array}\right\}
$$

where $\mu$ is the unique unstable eigenvalue for the Jacobian matrix $\boldsymbol{J}=\left.D_{x} \boldsymbol{f}(\boldsymbol{x})\right|_{\boldsymbol{x}=x_{e}}$ at $\boldsymbol{x}_{e}$.

Expanding the right side of equation (1) around the point $\boldsymbol{x}_{e}$, we obtain

$f(x)=f\left(x_{e}\right)+\boldsymbol{J} \cdot\left(\boldsymbol{x}-\boldsymbol{x}_{e}\right)+\left(\begin{array}{c}\left(\boldsymbol{x}-\boldsymbol{x}_{e}\right)^{T} \boldsymbol{H}_{1}\left(\boldsymbol{x}-\boldsymbol{x}_{e}\right) / 2 \\ \vdots \\ \left(\boldsymbol{x}-\boldsymbol{x}_{e}\right)^{T} \boldsymbol{H}_{m}\left(\boldsymbol{x}-\boldsymbol{x}_{e}\right) / 2\end{array}\right)+\cdots$

where $\boldsymbol{H}_{i}$ is the Hessian matrix of the function $f_{i}$ at the point $\boldsymbol{x}_{e}$, i.e. $\boldsymbol{H}_{i}=\left[\frac{\partial^{2} f_{i}}{\partial x_{k} \partial x_{l}}\right]_{m \times m}$. Then we can obtain the quadratic approximation for the stable manifold of the type- 1 equilibrium point $\boldsymbol{x}_{e}$ as following [22]

$$
h_{Q}(\boldsymbol{x})=\left[\boldsymbol{x}-\boldsymbol{x}_{e}\right]^{T} \boldsymbol{\eta}+\left[\boldsymbol{x}-\boldsymbol{x}_{e}\right]^{T} Q\left[\boldsymbol{x}-\boldsymbol{x}_{e}\right] / 2
$$

where $\boldsymbol{\eta}=\left(\eta_{1}, \cdots, \eta_{m}\right)^{T}$ is the left eigenvector for the Jocobian matrix, which satisfies

$$
\boldsymbol{J}^{T} \boldsymbol{\eta}=\mu \boldsymbol{\eta}
$$

and the quadratic coefficient $Q$ satisfies the following Lyapunov matrix equation

$$
C Q+Q C^{T}=H
$$

where the matrices $\boldsymbol{C}=\left(\mu \boldsymbol{I}_{m} / 2-\boldsymbol{J}^{T}\right), \boldsymbol{H}=\sum_{i=1}^{m} \eta_{i} \boldsymbol{H}_{i}$, $I_{m}$ is the $m \times m$ identical matrix.

There are a variety of methods for solving the Lyapunov matrix equation [23]. Two most efficient methods are the Schur method [24] and Hessenberg-Schur method [25]. The explicit unique solution of Lyapunov matrix equation (9) is

$$
\boldsymbol{Q}=V_{c}^{-1}\left[\left(\boldsymbol{C} \otimes \boldsymbol{I}_{m}+\boldsymbol{I}_{m} \otimes \boldsymbol{C}\right)^{-1} V_{c}(\boldsymbol{H})\right]
$$

where $V_{c}$ is the column stack mapping [26,p.5], which satisfies $V_{c}(\boldsymbol{B})=\left(b_{11}, \cdots, b_{m 1}, b_{12}, \cdots b_{m 2}, \cdots, b_{1 m} \cdots b_{m m}\right)^{T}$ for any the $m \times m$ square matrix $\boldsymbol{B}=\left(b_{i j}\right)_{m \times m}$, and $\otimes$ is the Kronecker tensor product, which satisfies $\boldsymbol{A} \otimes \boldsymbol{B}=\left(\begin{array}{ccc}a_{11} \boldsymbol{B} & \cdots & a_{1 t} \boldsymbol{B} \\ \vdots & \ddots & \vdots \\ a_{s 1} \boldsymbol{B} & \cdots & a_{s t} \boldsymbol{B}\end{array}\right)$ for any $s \times t$ matrix $\boldsymbol{A}=\left(a_{i j}\right)_{s \times t}$ and $k \times l$ matrix $\boldsymbol{B}=\left(b_{i j}\right)_{k \times l}$.

\section{Applying Quadratic Approximation to Multi- MACHINE SYSTEM}

We review the classical model for transient stability analysis. Consider a power system consisting of $n$ generators. Let the loads be modeled as constant impedances. Using the \#n machine as reference. Then dynamics of the $k$-th generator can be written with the usual notation as 
$\dot{\delta}_{k n}=f_{k}=\omega_{0} \omega_{k n}$

$\dot{\omega}_{k n}=f_{k+n-1}=-d_{0} \omega_{k n}+g_{k}\left(\delta_{1, n}, \cdots, \delta_{n-1, n}\right)$

$k=1, \cdots, n-1$

where $\quad g_{k}=\left(P_{m k}-P_{e k}\right) / 2 H_{k}-\left(P_{m n}-P_{e n}\right) / 2 H_{n}$

$d_{0}=D_{k} / 2 H_{k}(k=1, \cdots, n)$ is uniform damping. $D_{k}$ and

$H_{k}$ are damping ratio and machine inertia of machine $\# k$,

$P_{e k}=\left\{E_{k}^{2} G_{k k}+E_{k}\left(\sum_{j \neq k}^{n} E_{j}\left(G_{k j} \cos \delta_{k j}+B_{k j} \sin \delta_{k j}\right)\right)\right\}$ and $P_{m k}$

are the electrical and mechanical power at machine $\# k$, respectively. $\delta_{k j}=\delta_{k}-\delta_{j}, \delta_{k}$ is the rotor angle of machine \#k, $E_{k}$ is the constant voltage behind direct axis transient reactance of machine $\# k . \omega_{0}=2 \pi f_{B}, \boldsymbol{Y}=\left(G_{i j}+j B_{i j}\right)_{n \times n}$ is the reduced admittance matrix. For an $n$-generator system, the number of state variable is $2(n-1)$.

Let $\boldsymbol{x}=\left(\boldsymbol{\delta}^{T}, \boldsymbol{\omega}^{T}\right)^{T}=\left(\delta_{1, n}, \cdots, \delta_{n-1, n}, \omega_{1, n}, \cdots, \omega_{n-1, n}\right)^{T}$, $\boldsymbol{g}=\left(g_{1}, \cdots, g_{n-1}\right)^{T}$, and $\boldsymbol{J}_{0}=D_{\delta} \boldsymbol{g}$, then the Jacobian matrix at the equilibrium point $\left(\boldsymbol{\delta}_{0}^{T}, 0^{T}\right)^{T}$ of system $(11)$ is

$$
\boldsymbol{J}=\left[\begin{array}{cc}
\boldsymbol{0}_{(n-1)} & \omega_{0} \boldsymbol{I}_{n-1} \\
\boldsymbol{J}_{0} & -d_{0} \boldsymbol{I}_{n-1}
\end{array}\right]_{(2 n-2) \times(2 n-2)}
$$

where matrix $\boldsymbol{\theta}_{(n-1)}$ is the $(n-1) \times(n-1)$ matrix of zeros.

Assuming $\lambda_{0}$ is the unique unstable eigenvalue for matrix $\boldsymbol{J}_{0}$, and $\boldsymbol{\beta}_{0}$ is the corresponding left eigenvector, then the unique unstable eigenvalue $\mu$ of the matrix $J$ satisfies

$$
\mu=-d_{0} / 2+\sqrt{d_{0}^{2} / 4+\lambda_{0} \omega_{0}}
$$

and its corresponding left eigenvector is

$$
\boldsymbol{\eta}=\left[\begin{array}{l}
\boldsymbol{\alpha} \\
\boldsymbol{\beta}
\end{array}\right]=\frac{1}{\left(\sqrt{\left.\left(d_{0}+\mu\right)^{2}+\omega_{0}^{2}\right)}\right.}\left[\begin{array}{c}
\left(d_{0}+\mu\right) \boldsymbol{\beta}_{0} \\
\omega_{0} \boldsymbol{\beta}_{0}
\end{array}\right]
$$

Remark: Equation (13) and (14) implies that the unstable left eigenvector of the $(2 n-2) \times(2 n-2)$ matrix $J$ can be derived by the unstable left eigenvector of the $(n-1) \times(n-1)$ matrix $\boldsymbol{J}_{0}$.

At the CUEP, the Hessian matrices of $f_{k}$ are

$$
\begin{gathered}
\boldsymbol{H}_{k}=\boldsymbol{0}_{(2 n-2)} k=1, \cdots, n-1 \\
\boldsymbol{H}_{k+n-1}=\left[\begin{array}{cc}
N_{k} & \boldsymbol{0}_{(n-1)} \\
\boldsymbol{0}_{(n-1)} & \boldsymbol{0}_{(n-1)}
\end{array}\right]_{(2 n-2) \times(2 n-2)} k=1, \cdots, n-1
\end{gathered}
$$

$\boldsymbol{Q}=\left[\begin{array}{cc}\boldsymbol{Q}_{1} & \boldsymbol{Q}_{2} \\ \boldsymbol{Q}_{2}^{T} & \boldsymbol{Q}_{4}\end{array}\right]$, which satisfying the Lyapunov matrix equation (9), also satisfies the following equations

$$
\begin{gathered}
\boldsymbol{J}_{0}^{T} \boldsymbol{Q}_{2}^{T}+\boldsymbol{Q}_{2} \boldsymbol{J}_{0}-a \boldsymbol{Q}_{2}+b\left(\boldsymbol{J}_{0}^{T} \boldsymbol{Q}_{2}+\boldsymbol{J}_{0}^{T} \boldsymbol{Q}_{2}^{T}\right) \\
=-\sum_{i=1}^{k-1} \beta_{i} N_{i}=N_{0} \\
\boldsymbol{Q}_{4}=\omega_{0} /\left(2 d_{0}+\mu\right)\left(\boldsymbol{Q}_{2}+\boldsymbol{Q}_{2}^{T}\right) \\
\boldsymbol{Q}_{1}=\left[\left(d_{0}+\mu\right) \boldsymbol{Q}_{2}-J_{0}^{T} \boldsymbol{Q}_{4}\right] / \omega_{0}
\end{gathered}
$$

where $a=\mu\left(d_{0}+\mu\right) / \omega_{0}, b=\mu\left(2 d_{0}+\mu\right)$. The solution of equation (20) is

$$
\begin{aligned}
\boldsymbol{Q}_{2} & =V_{c}^{-1}\left\{\left[(1+b)\left(\boldsymbol{J}_{0}^{T} \otimes \boldsymbol{I}_{n-1}\right) \boldsymbol{W}_{[n-1]}+\boldsymbol{I}_{n-1} \otimes \boldsymbol{J}_{0}^{T}\right.\right. \\
& \left.\left.-a \boldsymbol{I}_{(n-1)^{2}}+b \boldsymbol{J}_{0}^{T} \otimes \boldsymbol{I}_{n-1}\right]^{-1} V_{c}\left(\boldsymbol{N}_{0}\right)\right\}
\end{aligned}
$$

where the matrix $W_{[n-1]}$ is the swap matrix ([26,p.5]), whose elements are zeros expect the elements at $(i+(j-1)(n-1), j+(i-1)(n-1)) \quad i, j=1, \cdots, n-1$; are all taking the value 1 .

Thus we obtain the quadratic approximation for the stability region of multi-machine power system with the $\operatorname{CUEP}\left(\left(\boldsymbol{\delta}^{e}\right)^{T},\left(\boldsymbol{\omega}^{e}\right)^{T}\right)^{T}$

$$
\begin{aligned}
h_{Q}(\boldsymbol{\delta}, \boldsymbol{\omega})= & \boldsymbol{\alpha}^{T}\left(\boldsymbol{\delta}-\boldsymbol{\delta}^{e}\right)+\boldsymbol{\beta}^{T}\left(\boldsymbol{\omega}-\boldsymbol{\omega}^{e}\right)+ \\
& \left(\boldsymbol{\delta}-\boldsymbol{\delta}^{e}\right)^{T} \boldsymbol{Q}_{1}\left(\boldsymbol{\delta}-\boldsymbol{\delta}^{e}\right) / 2+ \\
& \left(\boldsymbol{\delta}-\boldsymbol{\delta}^{e}\right)^{T} \boldsymbol{Q}_{2}\left(\boldsymbol{\omega}-\boldsymbol{\omega}^{e}\right)+ \\
& \left(\boldsymbol{\omega}-\boldsymbol{\omega}^{e}\right)^{T} \boldsymbol{Q}_{4}\left(\boldsymbol{\omega}-\boldsymbol{\omega}^{e}\right) / 2
\end{aligned}
$$

Once obtaining the quadratic approximation for the stability region of the post fault system we can estimate the CCT with the continuous fauted trajectory, i.e., identifying the time satisfying $h_{Q}(\delta(t), \omega(t))=0$, which is the time corresponds to the continuous trajectory intersecting with the quadratic approximation for the stable manifold.

\section{Simulation Results}

This section applies the proposed approach in section III to estimate the CCTs of faults in SMIB, IEEE 9-bus system and New England system.

A. Single Machine One Infinite Bus System

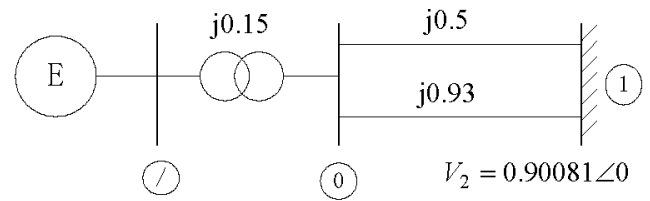

Fig. 1. Single-Machine-One-Infinite-Bus System (SMIB)

The approximation method proposed above has been applied to a SMIB system (Fig. 1.) Complete data for this system can be found in [27, p.844] except that the damping term with $D=0$ was added to the generator. The line 2 ( $X_{L 2}=0.93$ ) experiences a solid three-phase fault at the sending terminal of the high voltage side. The fault is cleared by isolating the faulted line. 


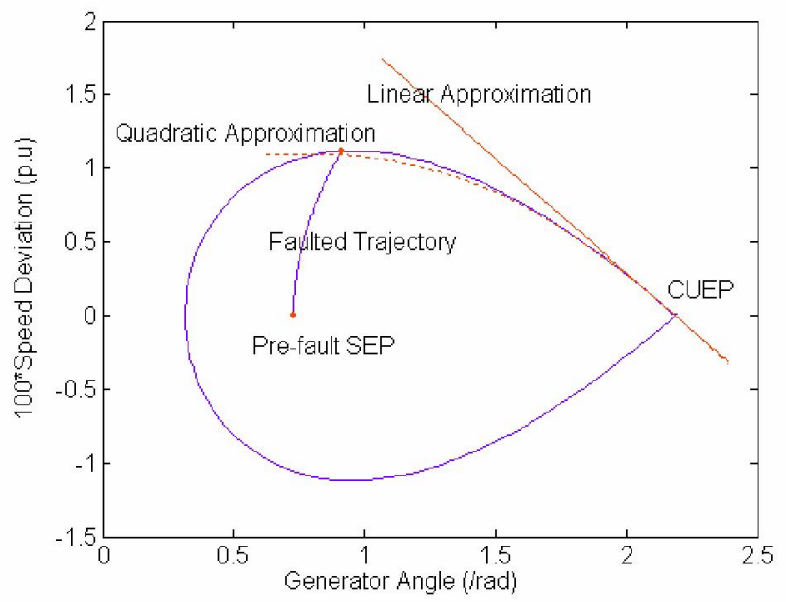

Fig.2 Mechanism of CCT Estimation by Stability Region Approximations

With the simulation time step being $0.001 \mathrm{~s}$, the time domain numerical integration method shows that the actual $\mathrm{CCT}$ is $0.086 \mathrm{~s}$, the quadratic approximation gives out a conservative estimation $0.084 \mathrm{~s}$, and the linear approximation gives out an over-estimated estimation $0.128 \mathrm{~s}$. Fig. 2 shows the mechanism of estimating $\mathrm{CCT}$ with the approximations for the stability region. In Fig.2, the continuous faulted trajectory intersects the approximated boundary of stability region at the estimated CCTs, the quadratic approximation is slightly conservative at the exit point, thus, its estimation is accurate while the linear approximation is seriously over-estimated and its estimation is poor.

\section{B. The 3-Generator 9-Bus System}

The simulation results in this subsection are based on a 3generator, 9-bus power system, which is an IEEE test system [28, p.38]. The system is showed in Fig.3. The types of faults are three-phase faults. The system are modeled by the network-reduction model with classical generators having the uniform damping $d_{0}=0.1661$. The time step adopted in the simulation is $0.0005 \mathrm{~s}$.

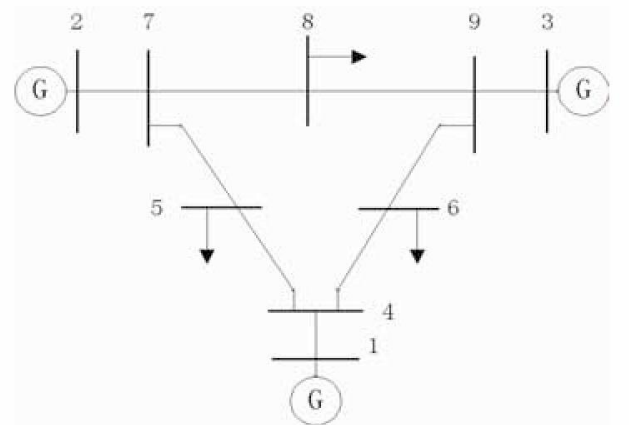

Fig.3 IEEE 3-Generator and 9-bus System.

Table I displays the estimated CCTs of six faults using five different methods: the time-domain numerical integration method (T-D), the quadratic and linear approximation of stability region, the BCU method [13] and the PEBS method [10]. The results from the time-domain numerical integration method are used as a benchmark. For example, the last row of table I states that a three phase fault occurs at bus 7 and the post fault system is the system with the transmission line between buses 7 and 5 tripped, due to the openings of circuit breakers at both ends of the line. The CCT estimated by the time domain simulation is $0.2025 \mathrm{~s}$, the CCT estimated by the quadratic approximation method is $0.1990 \mathrm{~s}$ and the CCT estimated by the linear approximation is $0.2595 \mathrm{~s}$, and the CCT estimated by the BCU and PEBS method is $0.2075 \mathrm{~s}$ and $0.2195 \mathrm{~s}$, respectively.

TABLE I

CCTS FOR FAULTS OF IEEE-9 BUS SYSTEM $\left(D_{0}=0.1661\right)$

\begin{tabular}{|c|c|c|c|c|c|c|c|c|c|}
\hline \multirow{3}{*}{$\frac{\text { Fault }}{(4) 4-6}$} & \multicolumn{9}{|c|}{ CCTs Estimated (Lnit: second) by different methods and Deviations } \\
\hline & \multirow{2}{*}{$\frac{\text { T-D }}{0.3555}$} & \multicolumn{2}{|c|}{ Quadratic } & \multicolumn{2}{|c|}{ Linear } & \multicolumn{2}{|c|}{$B C U$} & \multicolumn{2}{|c|}{ PEBS } \\
\hline & & 0.3895 & $104 \%$ & 0.3035 & $20 \cos \%$ & a 3515 & $-1,04 \%$ & 3540 & $-0.390 \%$ \\
\hline (4) 45 & 0.3865 & 0.3975 & $285 \%$ & 0.4020 & 4.016 & 01850 & $0.39 \%$ & 0.3860 & $0.13 \%$ \\
\hline (0)0-s & 0.2540 & 0.2670 & $5.12 \%$ & 0.3925 & $19.00 \%$ & 0.2315 & $-8.80 \%$ & 0.2000 & $5.91 \%$ \\
\hline gos & 0.2535 & 0.2595 & $2.37 \%$ & 0.2655 & $4.73 \%$ & 0.2305 & $5.71 \%$ & 0.2590 & $1.78 \%$ \\
\hline (7)7-8 & 0.2095 & 0.2155 & 2.8646 & 0.310 & $10.56 \%$ & 6.1830 & -1266 운 & 0.2230 & $6.44^{\phi}$, \\
\hline (7) 75 & 0.2025 & 0.1990 & $-1.73 \%$ & 0.2595 & $28.15 \%$ & 0.2045 & $0.99 \%$ & 0.2145 & $5.93 \%$ \\
\hline
\end{tabular}

Table I demonstrates that the proposed quadratic approximation method offers fairly accurate direct analysis of transient stability for these faults. The quadratic approximation gives out slightly over-estimated or conservative results in estimating $\mathrm{CCT}$, which is agree with the engineer requirements as BCU and PEBS methods, while the linear approximation gives out over-estimated and impractical results (which may exceed $28 \%$ ). This suggests that the quadratic approximation is also a good alternative to estimate the CCT.

\section{New England System}

The simulation results presented in this subsection are based on a 10-generator, 39-bus New England system [1]. The types of faults are three-phase faults with fault location both at generator and load buses. The system is modeled by the network-reduction model with classical generators having the uniform damping $d_{0}=0.2$. The time step adopted in the simulation is $0.001 \mathrm{~s}$.

Table II and Table III display the estimated CCTs of different faulted system using five different methods: the time-domain numerical integration method, the quadratic and linear approximation of stability region, the BCU method [13] and the PEBS method [10]. The results from the time-domain method are used as a benchmark.

TABLE II

CCTS FOR GENERATOR BUS FAULTS OF NEW ENGLAND SYSTEM

\begin{tabular}{|c|c|c|c|c|c|c|c|c|c|}
\hline \multirow{3}{*}{$\begin{array}{l}\text { Fault } \\
25) 25-26\end{array}$} & \multicolumn{9}{|c|}{ CCTs Estimated (Tnit: second) by different methods and Deviations } \\
\hline & \multirow{2}{*}{$\frac{\text { T.D }}{0.204}$} & \multicolumn{2}{|c|}{ Quadratic } & \multicolumn{2}{|c|}{ Linear } & \multicolumn{2}{|c|}{$\mathrm{BCU}$} & \multicolumn{2}{|c|}{ PEBS } \\
\hline & & 0.244 & $10.61 \%$ & 0.25 & $25+400$ & 188 & $-7,84 \%$ & 210 & 2.94 \\
\hline$(25) 25-2$ & 6.155 & 0.115 & $=11.2$ & 0.1 .9 & 8 & at. & $=21.1$ & 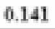 & $1 \%$ \\
\hline$(25) 23-23$ & 0.223 & 0.198 & -11.21 \% & 9.7 & 58 & 0.174 & -21.9 & 0.19 & -10.76 \\
\hline (10) $10-13$ & 0.242 & 0.249 & $2.390 \%$ & 0.324 & $33.58 \%$ & $0.25 s$ & $6.61 \%$ & 0.215 & $-11.15 \%$ \\
\hline (10)10-11 & 0.245 & 0.256 & $4.490 \%$ & 0.349 & tous & 0.25 & 2.4506 & 0.224 & 9.5756 \\
\hline 1$) 21-22$ & 0.167 & 0.148 & $-11.35 \%$ & 0.308 & $8+43^{2} 6$ & 0.224 & $34,13 \%$ & 0.139 & -16.77 \\
\hline
\end{tabular}

Table II demonstrates that the proposed quadratic approximation method offers not quite accurate direct analysis of transient stability for some faults at the generation buses. 
The deviations of the CCTs obtained by the quadratic approximation method may be $20 \%$ or so, and the deviations of the CCT obtained by the linear approximation method are too serious to use. This suggests that the quadratic approximation may be not a good alternative to estimate the CCTs of the faults located at generator buses.

$$
\text { TABLE III }
$$

CCTS FOR NON-GENERATOR BUS FAtLTS OF NEW ENGLAND SYSTEM

\begin{tabular}{|c|c|c|c|c|c|c|c|c|c|}
\hline \multirow{3}{*}{$\begin{array}{l}\text { Fault } \\
\text { (3) } 3-4\end{array}$} & \multicolumn{9}{|c|}{ Hed (C Din: secosd) } \\
\hline & \multirow{2}{*}{$\frac{\text { T.D }}{0.266}$} & \multicolumn{2}{|c|}{ Quadratic } & \multicolumn{2}{|c|}{ Linear } & \multicolumn{2}{|c|}{$\mathrm{BCU}$} & \multicolumn{2}{|c|}{ PEBS } \\
\hline & & 0.280 & $5.26 \%$ & & 1.31 & al & $1.85 \%$ & $125 \mathrm{~s}$ & -3.0 \\
\hline (5) 56 & 243 & 0.2 & & & & & & 0.222 & $\$ 6$ \\
\hline & & & & & & & & $12+2+3$ & 3. \\
\hline & 247 & & & & & 0.274 & 0.9. & 9 & 0.4 \\
\hline & 0.05 & & .54 & & & & & ow & 2 \\
\hline 129-28 & 0.061 & 0.057 & $-6.5 \% \%$ & Q.M & 81.974 & 0.051 & $16.39 \%$ & 0.51 & 16.3 \\
\hline
\end{tabular}

Table III demonstrates that the proposed quadratic approximation method offers fairly accurate direct analysis of transient stability for faults located at non-generator buses. The quadratic approximation gives out slightly conservative or over-estimated results in estimating CCT, the deviations are about $5 \%$, while the linear approximation gives out much more over-estimated results (deviations range form $20 \%$ to $80 \%$ ), and the BCU and PEBS methods may give out results with great large deviation. This suggests that the quadratic approximation method is a good alternative to estimate the CCTs of the faults located at non-generator buses.

\section{Conclusions}

This paper applies the quadratic approximation of the stable manifold to approximate the boundary of stability region for the multi-machine power systems with networkreduced model, furthermore calculates the CCT. In obtaining the coefficients of quadratic approximation, we explore the special structure of the model and develop the decomposition method to reduce the computation. The CCT is estimated by the time corresponds to the continuous trajectory intersecting with the quadratic surface approximation for the stable manifold. The simulations in the 9-bus system and New England system show that the proposed approach could give out fairly good estimation for the CCT of median-size power system, while the simulation results in large-scale systems are still need to be developed.

\section{REFERENCES}

[1] M. A. Pai, Energy function analysis for power system stability. Boston: Kluwer Academic Publishers, 1989.

[2] A. A. Fouad, V. Vittal. Power system transient stability analysis using the transient energy function method, Englewood Cliffs, N. J. : Prentice Hall, 1992.

[3] M.Ribbens-Pavella and P.G. Murthy, Transient Stability of Power systems: Theory and Practice, New York: Wiley, 1994

[4] H. D. Chiang, C. C. Chu, and G. Cauley, "Direct Stability Analysis of Electric Power Systems Using Energy Functions: Theory, Applications and perspective", Proceedings of the IEEE, Vol. 83, No.11, pp.1497 1529, Nov. 1995.

[5] S. Liu, J. Wang, Energy Function Analysis for Power System Transient Stability, Shanghai: Shanghai Jiaotong University Press. 1996. (In Chinese)
[6] Y. X. Yu, C. S. Wang, Theories and Methods of Electrical Power System Stability, Beijing: Science Press. 1999. (In Chinese)

[7] H. D. Chiang, M. W. Hirsch, F. F. Wu, "Stability regions of nonlinear autonomous dynamical systems", IEEE Transactions on Automatic Control, Vol. 33, No. 1, pp.16-27, Jan. 1988.

[8] H. D. Chiang, J. S. Thorp, "The closest unstable equilibrium point method for power system dynamic security assessment", IEEE Transactions on Circuits and Systems, Vol. 36, No. 9, pp. 1187-1200, Sep. 1989.

[9] H. D. Chiang, F. F. Wu, P. P. Varaiya, "Foundation of direct methods for power systems transient stability analysis", IEEE Transactions on Circuits and systems, Vol. CAS-34, No.2, pp.160-173, Feb. 1987.

[10] N. Kakimoto, Y.Ohsawa and M. Hayashi, "Transient stability analysis of Electric-Power System via Lure-Lypunov function, Part I and II", Trans, IEE Japan, Vol. 98, No.5/6, 1978.

[11] T. Athay, R. Podmore and S. Virmani, "A practical method for directed anaysis of transient stability", IEEE trans. On Appar.\&Syst., Vol. PAS98, No. 2, 1979.

[12] H. D. Chiang, F. F. Wu, P. P. Varaiya, "Foundations of the potential energy boundary surface method for power system transient stability analysis", IEEE Transactions on Circuits and Systems, Vol. 35, No. 6, pp.712-728. June 1988.

[13] H. D. Chiang, C. C. Chu, "Theoretical foundation of the BCU method for direct stability analysis of network reduction power system models with small transfer conductance", IEEE Transactions on Circuits and Systems I: Fundamental Theory and Applications, Vol. 42, No. 5, pp. 252-265, May 1995.

[14] H. Yee and B. D. Spading, "Transient stability analysis of multi-machine systems by the Method of Hyperplances", IEEE Transactions on PAS, Vol. PAS-96, pp. 276-284, 1977.

[15] P. A. Cook and A. M. Eskicioglu, "Transient stability analysis of electric power systems by the method of tangent hypersurface", IEE Proceedings. Part C, Vol. 130, pp. 183-193, July 1983.

[16] Y. Min, H. Li, Y. J. Liu, "Critical fault clearing time of multi machine system with the quadratic surface method", Journal of Tsinghua University (Secience and Technology), Vol. 44, No. 4, pp. 454-457, 2004.

[17] S. Ushiki, "Analytic expression of the unstable manifolds," Proc. Japan Acad. 56, Ser. A, pp. 239-244, 1980.

[18] F. M. S. Faithi, A. Arapostathis, and P. P. Varaiya, "Analytic expressions for the unstable manifold at equilibrium point in dynamical systems of differential equations," Proceedings of the $22^{\text {nd }}$ IEEE Conference on Decision and Control, Vol. 3, pp. 1389-1392, 1983.

[19] S. Saha, A. A. Fouad, W. H. Kliemann, V. Vittal, "Stability boundary approximation of a power system using the real normal form of vector fields," IEEE Transactions on Power Systems, Vol. 12, No. 2, pp.797802, May 1997.

[20] V. Venkatasubramanian, Weijun JI, "Numerical Approximation of (n-1) dimension stable manifold in large systems such as power systems," Automatica, Vol. 33, No. 10, pp. 1877-83, 1997.

[21] D. Z Cheng, J. Ma, "Calculation of stability region," in Proceedings. 2003 (42nd) IEEE Conference on Decision and Conirol, Vol. 6, pp $5615-5620,2003$

[22] D. Z. Cheng, J. Ma, Q. Lu, S. W. Mei, "Quadratic form of stable submanifold for power systems", International Journal of Robust and Nonlinear Control, Vol. 14, Issue: 9-10, pp.773-788, 2004

[23] B. N. Datta, "Numerical Linear Algebra and Applications" . Brooks Cole Publishing Co., Pacific Grove, California, 1995. (Chapeter 8: Numerical solutions and Conditioning of Lyapunov and Sylyester equations.)

[24] R. H. Bartels and G.W. Stewart, "Algortihm 432: Solution of the Matrix Equation $A X+X B=C$," Communcation of the $A C M$, Vol. 15, No. 9, pp.820-826, 1972.

[25] G. H. Golub, S. Hash, and C. Van Loan, " A Hessenberg-Schur Methods for the problem $A X+X B=C$," IEEE Transactions on Automatic Control, Vol.24, No.6, pp. 909-913, 1979.

[26] D. Z. Cheng, Matrix and Polynomial Approach to Dynamic Control System, Beijing: Science Press, 2002.

[27] P. Kundur, Power System Stability and Control, New York: Mc-GrawHill, 1992.

[28] P. M. Anderson and A. A. Fouad, Power System Control and Stability, The Iowa State University Press, Ames, Iowa, U.S.A. 1977. (Revised Printing, IEEE Press, 1994. ( p375)) 


\section{BIOGRAPHIES}

Ancheng Xue was born in Jiangsu, China, in Apr. 1979. He received his B. Sc degree in applied mathematics from the Department of Mathematics, Tsinghua University. He is currently a Ph. D candidate in the Department of Electrical Engineering, Tsinghua University. His research interests include the nonlinear systems, control theory and their applications to the power systems.

Felix F. Wu (SM'86, F' 89 ) received his $\mathrm{Ph}$. D from University of California at Berkeley (UCB). He is currently Philip Wong Wilson Wong Professor in Electrical Engineering, at the University of Hong Kong, and Professor Emeritus, Department of Electrical Engineering and Computer Sciences at University of California, Berkeley.

Yixin Ni (SM'94) received her B. Eng., M. Eng., and Dr. Eng. All from the Department of Electrical Engineering. Tsinghua University, China in 1968 1981 and 1983 respectively. Her research interests are power system stability and control, HVDC transmission, FACTS, and electricity markets. She was a professor of Tsinghua University and is now with the University of Hong Kong. She is a recipient of several nation-wide awards.

Qiang Lu (SM'85, F'02) graduated from the Graduate School of Tsinghua University, China, in 1963 and joined the faculty of the same University. He was a visiting scholar and a visiting professor in Washington University, St. Louis and Colorado State University, Ft. Collins, respectively in 1984-1986 and a1993-1995. He is now a professor in Tsinghua University, and an academician of Chinese Academy of Science (1991). His research interest is in nonlinear control theory applications in power systems.

Shengwei Mei (M'99) was born in Xinjiang, China, on Sept. 20, 1964. He received his B.S. degree in mathematics from Xinjiang University, M.S. degree in operations research from Tsinghua University, and $\mathrm{Ph} . \mathrm{D}$. degree in automatic control from Chinese Academy of Sciences, Beijing, in 1984, 1989, and 1996 respectively. He is now a professor of Tsinghua University. His current interest is in control theory applications in power systems. 